\title{
Entities and Relations for Agent-Based Modelling of Complex Spatial Systems
}

\author{
Pedro Ribeiro de Andrade ${ }^{1}$, Antônio Miguel Vieira Monteiro ${ }^{1}$, Gilberto Câmara ${ }^{1}$ \\ ${ }^{1}$ Image Processing Division (DPI) \\ National Institute for Space Research (INPE) \\ \{pedro, miguel, gilberto\}@dpi.inpe.br
}

\begin{abstract}
This work presents a contribution towards generalizing the representation of geospatial entities and their relations for simulating complex spatial systems using the agent-based approach. We analyse the works in the literature, and argue that each of the four types of relation is necessary. These relations can be grouped in two classes, placements and neighbourhoods, with likenesses and differences between them. Given that, we define requirements for representing geospatial entities and their relations, and study six toolkits for ABM (Netlogo, OBEUS, Repast, Swarm, GRSP, and TerraME), analysing their capabilities to address the proposed requirements. Finally, we present our current work and future directions on developing the TerraME toolkit.
\end{abstract}

Keywords: ABM, GIS, toolkits, requirements.

\section{Introduction}

The human being has always changed its environment. Changes can occur at the physical, social, and economic levels, ranging from deforestation to global warming, from individual mobility to seggregation patterns, or from an ordinary bargaining between buyer and seller to an economic chain dynamics, to name a few. They result from complex interactions among the entities directly and indirectly involved in the system. The inherent complexity of the processes involved in the system shows nonlinearity, displayed by a complex behaviour that presents thresholds, positive and negative feedback loops, lags in time and space, resilience, heterogeneity, and, above all, surprises [1].

The living earth is the natural support where complex relations evolve over time. It has great influence in our decision-making process, and in how we set up and maintain relations, because we are grounded to places and regions with unique characteristics and dynamics. Although some production factors such as capital, labour, or even information are less spatially sensitive (at least in developed places), natural resources and consumers are where they are. Moreover, even though the newest communication technologies promise to ignore the impact of distance on human relations [2], proximity remains crucial in effective collaboration, and the technological development mitigates only part of the negative impacts of distance on productivity [3]. 
With the development of technologies for acquisition and manipulation of data, especially geospatial data, it is now possible to work with models at individual level. It allows building up models with a higher disaggregation, which allows considering the modifiable areal unit problem. Different methods for data acquisition are available, such as GPS and remotely sensed data, surveys, participant observation, and field and laboratory experiments. These methods can be combined to increase the model effectiveness [4], but the main difficulty stands in how to manipulate the diversity of data provided by these methods.

In the past, researchers have used Geographic Information Systems (GIS) to study these interactions. But, although GIS have strong functionalities to manipulate and to analyse geographical data, it is hard to model temporal dynamics at individual level within GIS environments because they are based on data models, instead of process models [5]. Usually, GIS with embedded dynamical predictive models are built as customized tools and work only with a specific process [6]. In fact, most of them are created with physical rather than human processes in mind. The only way to model social or economic problems is thinking in human behaviour as if it was physical. For example, applications that use Cellular Automata (CA) for studying human behaviour anthropomorphize the state variables of a cell.

Agent-Based Modelling (ABM) comes as an approach for addressing these issues broadly. ABM provides a bottom-up method for studying complex systems through the dynamical interaction of agents. It allows representing individual behaviour and cognitive process, which cannot be fully attained by approaches such as mathematical or statistical modelling. Although some disbelief has taken place on this approach [79], it has been shown that ABM can unquestionably increase our understanding of human behaviour [10-13].

Newest software toolkits have made ABM easy enough to be attractive to areas such as economics, sociology, anthropology, physics, and biology. The use of simulation toolkits relieves the modeller of programming the parts of the simulation that are not content-specific, such as simulation control and data structures [14-16]. It also increases the reliability and efficiency of the model, as the most complex parts have been created and optimised by professional developers.

Recently, agent-based models have begun to use real-world spatial data for studying complex spatial systems [17]. However, most of the toolkits were initially created to work with landscapes that do not consider the environment in which we move and interact. They rely on non-grounded agents, in the sense that they do not represent entities which can sense and act in a representation of the real world. To follow the current development, the toolkits have to carry out the support for working with spatial data.

This work presents a contribution towards generalizing the representation of geospatial entities and their relations for simulating complex spatial systems using the agent-based approach. We revise and analyse the works in the literature, and define requirements for representing geospatial entities and their relations. We focus on the geographic needs - for general requisites there is already a couple of works $[14,16$, $18,19]$. We analyse some toolkits for ABM and their abilities to address the proposed requirements. Finally, we present our current work, towards fulfilling these requirements in the TerraME toolkit. 


\section{Reviewing the entities and their relations}

In this section we present the works in the literature about the relations between the entities of agent-based models for complex spatial systems. Before defining their relations, we need to introduce the entities of a system, which belong to a scale.

\subsection{Scale and its components}

Gibson and others review the idea of scale in the literature of social science, especially related to the human dimensions of global environmental change [20]. The causes and outcomes of the human actions can be measured at different levels along multiple scales. Scale is a well-defined idea in the natural sciences, but it has fuzzy borders in the social sciences. Nevertheless, the authors show that each scale has three dimensions: spatial, temporal, and behavioural (analytical). In this work, we are interested in computer simulations; therefore each dimension has to be discretized in some way.

\subsubsection{The spatial component of a scale}

The spatial data has to be divided into single partitions, called cells, whose internal content is homogeneous, in such a way that any change in a cell affects all its extension. With it, it is possible to change each cell independently from the others, which allows working with heterogeneous data.

GIS work with a partitioned space, be it a vector or a raster data. Dividing the space in rectangular entities has advantages over the vectorial representation because it simplifies the representation and helps creating neighbourhood relations [21]. But vector data can bring more reality, mainly when the spatial data represents some economic, political, or social convention.

\subsubsection{The behavioural component of a scale}

Instead of using a set of equations for describing the behaviour of a model in an aggregated way, the behavioural entities are described as agents. Each agent can affect itself, the space, and the other agents, and the interactions between the agents can produce an emergent pattern. Modelling with agents allows a more aggressive exploration of characteristics such as imperfect rationality, social and institutional structures, and distinct behaviours in different locations.

Agents can represent geospatial entities such as farmers or householders. Therefore, just like for representing the space, they can be associated to objects stored in a geographic database, whose information layer contains the spatial representation of the agents as well as their properties. Although there are some advances in visual languages, the behaviour of agents and a complementary part of their properties still have to be described in a programming language outside the GIS environment.

\subsubsection{The temporal component of a scale}

Each entity of a model can have a frequency in which it decides its actions. There are two choices for describing temporal events. The first is synchronous (parallel) 
execution, where each agent is an independent process. The advantage of this approach is that the time one agent takes for its decision-making is always considered. Thus, agents that need more time to compute their decisions are in disadvantage. The main problem of this approach is that the modeller has to give some attention to avoid temporal conflicts, which can significantly increase the complexity of the model.

The second alternative is asynchronous (sequential) execution, with the time discretized as a scheduler of actions. As an agent usually takes much more time to perform changes in the geographic space than to compute its decisions, we can usually ignore the processing time. Discrete time allows a straightforward solution to any temporal conflict between agents, defining a predetermined history of events to take place along the execution. It simplifies replicating models because the agents do not work in parallel. Also, once there is not any conflict between the actions of the agents, it is possible to simulate every parallel step using two sequential steps.

As each agent has its own frequency of execution, it is possible to have events with different temporal frequencies, or even events occurring only once. The capacity to schedule events at different times allows the modeller to simulate the temporal aspects of the specific urban problem under study [22].

\subsection{Relations following Torrens and Benenson}

In each step of its execution, a model is at a given time, which always increases. Once the scheduler finishes all the events of a given time, it increments the time and then start processing the events of the next time. The time entity is conceptually different from the other two because their extents are completely available at each time step. Therefore, we have agents and cells as the two main entities of a spatial phenomenon at a given scale, while the time is in charge of the execution flow.

A relation is a mapping that describes the connection between two entities. Combining the two classes of entities, we have four types of relations: cell $\rightarrow$ cell, agent $\rightarrow$ cell, agent $\rightarrow$ agent and cell $\rightarrow$ agent. Torrens and Benenson use a leader and follower approach to define the spatial relations [23]. The leader is responsible for managing the relation, while the follower is a passive object. The aim of the authors is to keep consistency in the relations, avoiding conflicts between the entities:

"[...] an application of the [...] rules that describe these changes [on the relations] might cause conflicts, when, in housing applications, for example, a landlord wants to sell his property, while the tenant does not want to leave the apartment. Who has the right to destroy the relationship between the tenant and the property, then? This example represents the general problem of consistency in managing relationships."

Following the leader and follower idea, we have agents as leaders and cells as followers. The relation cell $\rightarrow$ cell has two followers; therefore it is a static representation, storing spatial neighbourhoods. Agent $\rightarrow$ cell specifies which cells one agent has or controls. The agents are free to manage this relation.

The two other relations do not fit in the leader and follower definition. One cell cannot be a leader in a cell $\rightarrow$ agent. The authors solve this relation by using a backward query in agent $\rightarrow$ cell. Agent $\rightarrow$ agent is not feasible because it is not possible to define the leader of the relation. The authors argue that the only way two agents can communicate is by using the other available relations in a transitive way 
(agent $\rightarrow$ cell, then cell $\rightarrow$ cell, then cell $\rightarrow$ agent). Moreover, in their toolkit User's Guide, Benenson and Harbash argue that "this limitation might be inconvenient if you develop psychological model, but, luckily, we model not love affairs, but the collective urban spatial phenomena" [24]. Table 1 summarises the four relations and how the authors solve each one. The authors call the relations between two entities of the same type (agent $\rightarrow$ agent and cell $\rightarrow$ cell) as neighbourhood, and the other two as geo-referencing. They consider the relations as being binary, that is, at each time, two entities are either connected or not.

Table 1: Cell and agent relations, according to [23].

\begin{tabular}{|c|l|l|}
\hline From & \multicolumn{1}{|c|}{ Cell } & \multicolumn{1}{c|}{ Agent } \\
\hline Cell & $\begin{array}{l}\text { Neighbourood relation } \\
\text { (static) }\end{array}$ & $\begin{array}{l}\text { Agents who are in a certain } \\
\text { cell (backward query) }\end{array}$ \\
\hline Agent & $\begin{array}{l}\text { Cells an agent } \\
\text { controls (dynamic) }\end{array}$ & $\begin{array}{l}\text { Prohibited relation } \\
\text { (transition) }\end{array}$ \\
\hline
\end{tabular}

\section{A critical analysis of the leader and follower approach and a new proposal}

Torrens and Benenson cite an example of urban conflict, to which the leader and follower approach can solve the consistency problem shown in the last section:

"Accounting for limitations of OBEUS (direct relationships between agents are not allowed) the way to force the tenant to leave the property is to raise their payment. The tenant (the leader in tenant-property relationship) will likely end the relationship by herself in that case."

Even accounting the limits of this approach, the authors argue that

" $[\mathrm{t}]$ here is no proof that the majority of real-world situations can be imitated by the leader-follower pattern, although we are not aware of any natural instance where this pattern is insufficient."

The point on their example is that, in a real world situation, the landowner cannot one-sidedly increase the payment to force the tenant to leave, because this relation is often formally settled by a location contract. The only legal way to the landowner force the tenant to leave the property is by breaking the contract, accepting the outcomes imposed by its clauses. For that, we would need another agent with the power to break the relation, even without the tenant agreement. Therefore, the leader and follower approach cannot be used in this example.

Even if we consider true the statement that raising the payment will force the tenant to leave the property, we would need to assume that the seller owns all the rights on the contract, except the right to cancel it. But any toolkit that intends to have a generalized representation of the spatial entities and their relations cannot be limited to any a priori statement. Therefore, guarantee consistency in the relations has to be part of the model, instead of a restriction of the toolkit, because there can be another model that needs a different consistency in the relations.

The proposal of having agent $\rightarrow$ agent relations only in a strictly transitive way is another limitation of using the leader and follower approach. Patterns of change may 
arise locally, but social structures do not have to, and the technological development makes it even more fragmented. In fact, there are relations which do not rely on geographical closeness.

Instead of trying to prove that this or that relation is not necessary in a modelling toolkit, we propose that every relation type is necessary. Whenever there is a conflict over changing or not some relation, it has to be implemented in the model. The ways to solve conflicts can be based on the idea of autonomy proposed by Barber and Martin [25]. They argue that:

"[a]n agent's degree of autonomy [...] is the degree to which the decisionmaking process, used to determine how that goal [of the agent] should be pursued, is free from intervention by any other agent."

Conflicts can be solved by an agreement, when the agents follow a true consensus, or by a superior instance with more autonomy, called supervisor. One agent is locally autonomous only when no other agent can affect its decision-making. Barber and Martin use the term locally autonomous instead of just autonomous because they consider one agent may have different levels of autonomy for different goals.

We divide the relations in two groups: placement and neighbourhood. Following we describe each one, expose the reasons of this classification, and argue that each relation is necessary, presenting examples of works that identify or use them.

\subsection{Placement relations}

Each relation involving two entities of distinct types is a placement. Agent $\rightarrow$ cell and cell $\rightarrow$ agent represent the relations of this group. Cell $\rightarrow$ agent stores the agents that belong to a cell. Each cell may have one or more agents within it, and one agent can be at most in one place at any given time. One can easily implement movement on the space by changing this relation. This is the most common representation available in toolkits because there are many models which use it, for example [13].

In the other side, agent $\rightarrow$ cell represents the cells one agent controls, targets, watches, or any other objective depending on the context. This representation has a growing use in studies on land-use, where one agent represents a householder which has to choose what to do with its own territory. As example we can cite [26].

Two points are similar in placement relations. They are binary relations because, at any given time, two entities are either connected or not. One agent controls or not a cell; one agent is within or without a cell. Placement relations are also symmetric; if an agent is within a given cell then the cell contains the agent, and conversely. The same rationale is valid for the agent $\rightarrow$ cell relation. Therefore we agree with Torrens and Benenson in these relations. But we changed the term geo-referencing to placement because we are working with topological relations, and geo-referencing is closer to geometry than to topology.

\subsection{Neighbourhood relations}

Each relation involving two entities of the same type is a neighbourhood. We have two cases: cell $\rightarrow$ cell and agent $\rightarrow$ agent. A neighbourhood between cells represents their spatial proximity. Common representations include the well-known Euclidean 
neighbourhoods, Moore and von Neumann, which represent a basic definition of proximity: adjacency. But the geographic space by itself is not restricted to Euclidean spaces. Natural features such as mountains and rivers may affect distances, and the human action has significantly contributed for changing proximity relations. Neighbourhoods can be created from metrics such as distance, visibility, or accessibility. The result of these metrics can be in the form of weights, suggesting strengths to the connections between cells.

The connection between two agents is often called link, or tie. A link can be based on the time, the emotional intensity, the intimacy (mutual confiding), and the reciprocal services two agents spend with each other [27]. A recent work shows that spatial proximity remains crucial in effective collaboration, and the technological development mitigates only part of the negative impacts of distance on productivity [3]. Moreover, geography remains crucial even in on-line friendships. A study in a blog network shows that geographic processes draw nearly $70 \%$ of on-line friendships [28], but it does not mean the other $30 \%$ results only from non-spatial processes (the Internet, in this case).

A set of interconnected entities, be they agents or cells, form a network. From a network we can extract metrics that describe the whole composition of the network, providing signatures to compare it with other networks, or even for creating synthetic networks. Geospatial networks can be grouped in classes with strong signatures, distinct from one another and from non-geographic networks [29]. Social networks differ from spatial networks (such as road networks) because they are divided into communities, presenting higher levels of clustering [30]. Most agent-based simulations use only basic network arrangements in arbitrary ways or as a computational criteria, but "it seems likely that network topology should have some (and as yet unknown) effect on resulting processes and emergent behaviour" [31].

A clear example of using agent $\rightarrow$ agent relation for building networks for complex spatial systems is in chain models. In these models, we have groups of agents with connections within and between groups. For instance, agents may connect to others according to economic interests to minimize production and transport costs. Recently, we have seen the food crisis that has feared the world because of its potential to cause social and economic problems. One of the ways to bypass this problem is exploring the whole economic chain, trying to identify the weaknesses of its nodes and add more value to them.

Differently from placements, neighbourhoods are not boolean, but weighted. The weight of each neighbourhood points out the strength of the connection between the entities. Also, neighbourhoods are not symmetrical, but directed. It is possible to have A connected to B with a weight different from B to A. Note that symmetric networks are a subset of directed networks, therefore we can have symmetric neighbourhoods, but not directed placements. Therefore, our approach differs to the proposal of Torrens and Benenson in this case for both characteriscs: directed and weighted. Table 1 summarizes the two types of relation of our approach. 
Table 1: Relations and their differences

\begin{tabular}{|l|c|c|c|c|}
\hline \multirow{2}{*}{ Flow } & \multicolumn{2}{|c|}{ Neighbourhood } & \multicolumn{2}{c|}{ Placement } \\
\cline { 2 - 5 } & Information & Cells & Agents & Cells \\
\hline Type & Weighted & Weighted & Binary & Binary \\
\hline Graph & Directed & Directed & Symmetrical & Symmetrical \\
\hline Weight & $\begin{array}{c}\text { Amount of time, intimacy } \\
\text { emotional intensity, } \\
\text { reciprocal services, }\end{array}$ & $\begin{array}{c}\text { Adjacency, contiguity, } \\
\text { continuity, distance, } \\
\text { accessibility, visibility }\end{array}$ & - & - \\
\hline
\end{tabular}

\section{Requirements for simulating complex spatial systems}

Simulating complex spatial systems using ABM needs GIS representations for both entities and their relations. The requirements related to the relations are twofold: create and manipulate them. The initial arrangement of each relation can be created within a GIS and loaded from the database to the model. When the relations are previously stored in a database, it is possible to reduce the simulation time whenever we need to replicate the model to verify its convergence, because create some of these relations can be time demanding.

The requirements related to the entities are:

Space. The integration between a toolkit and a GIS should support reading geographic data and their properties, in such a way that the objects of a layer of information have a one-to-one mapping to the spatial objects of the model.

Behaviour. As well as for representing the space, agents can be associated to objects stored in geographic databases, with a spatial location and properties. As GIS work with spatial data in an object-oriented way, agents within a given layer have the same properties and the same geometric type.

Time. Besides the scheduler, temporal data that is independent of the behavioural description of the model, usually exogenous events such as building a new road, has to be manipulated by the temporal entity when the data belongs to a geospatial database. The temporal data available in the database has to be automatically loaded before any behaviour take place at any given time. Temporal changes can be data from the space, the agents, or the relations. The modeller only has to keep in mind which properties the agents can change, and those which are going to be automatically read from the database, if any.

\section{$5 \quad$ Toolkits for agent-based modelling}

In this section, we present the most popular toolkits for ABM and their extensions for working with complex spatial systems. We analyse if they support the requirements described in last section. The toolkits are Swarm [32, 33], Repast [34, 35], OBEUS [23], and Netlogo [36]. We also study two toolkits developed by Brazilian institutes: GRSP [37] and TerraME [38]. Due to space constraints, we do not present the toolkits individually, but a general description of each can be found in their references.

Every toolkit can import some geographic data for representing the space, but only some of them support agents as geospatial entities. Swarm and Netlogo can load raster data for representing space, in ASCII and PPM (Portable Pix Map) formats, 
respectively. Repast, OBEUS, and GRSP can represent both space and agents as geospatial entities. Repast uses shapefiles for vector data and ASCII for raster data, OBEUS is a GIS whose objects can change their properties and locations in time, and GRSP accesses PostgreSQL databases. TerraME works with cellular spaces, which are rectangular grids with properties created from raster or vector data stored in TerraLib databases [39], but it cannot represent agents with geospatial data. None of the analysed toolkits have any control over temporal databases.

In the side of the relations, the most common representations available are placement of agents into cells and neighbourhood of cells, but the toolkits usually do not go further than Euclidean proximities. Exceptions are OBEUS, Repast and TerraME. OBEUS and Repast can use complex vector operators such as point-inpolygon, buffering and intersection to calculate the proximity between cells. OBEUS uses the leader and follower approach to get agent $\rightarrow$ agent transitively from the other relations. TerraME uses a more general idea of non-proximal spaces. It can access complex neighbourhoods created not only by the relations between two objects, but it can also use a third component, for instance a transport network, to calculate proximity. It is called Generalized Proximity Matrix, or GPM [40]. But, as TerraME does not represent agents as geospatial entities, there is no way to create neighbourhoods for agents.

The lack of complex neighbourhoods in toolkits is due to the difficulty to create complex relations from scratch, which can be easily built within a GIS environment. Moreover, it seems that agent $\rightarrow$ agent relation is commonly left aside because this is the only of the four relations that does not have any direct relation to the geographic space. Probably that is why Torrens and Benenson resolve this relation using the other relations to make the use of the geographic space explicit. Table 2 summarizes the capacities of the analysed toolkits.

Table 2: Comparison of the toolkits for working with geospatial data

\begin{tabular}{|l|c|c|c|c|c|c|c|}
\hline \multirow{2}{*}{ Toolkit } & Space & \multirow{2}{*}{ Time } & \multirow{2}{*}{ Agents } & \multicolumn{2}{|c|}{ Neighbourhood } & \multicolumn{2}{|c|}{ Placement } \\
\cline { 5 - 8 } & & Agent & Cell & Agent $\rightarrow$ Cell & Cell $\rightarrow$ Agent \\
\hline Netlogo & Raster & $*$ & $*$ & $*$ & Euclidean & Manipulate & $*$ \\
data & $*$ & $\begin{array}{c}\text { Vector } \\
\text { data }\end{array}$ & $*$ & Euclidean & $\begin{array}{c}\text { Manipulate, } \\
\text { create }\end{array}$ & $*$ \\
\hline OBEUS & $\begin{array}{c}\text { Vector } \\
\text { data }\end{array}$ & $*$ & $\begin{array}{c}\text { Vector } \\
\text { data }\end{array}$ & $\begin{array}{c}\text { Transi- } \\
\text { tion }\end{array}$ & $\begin{array}{c}\text { Complex } \\
\text { operators }\end{array}$ & $\begin{array}{c}\text { Manipulate, } \\
\text { create }\end{array}$ & $\begin{array}{c}\text { Manipulate, } \\
\text { create }\end{array}$ \\
\hline Swarm & Raster & $*$ & $\begin{array}{c}\text { Vector } \\
\text { data }\end{array}$ & $*$ & $\begin{array}{c}\text { Complex } \\
\text { operators }\end{array}$ & $\begin{array}{c}\text { Manipulate, } \\
\text { create }\end{array}$ & $*$ \\
\hline TerraME & $\begin{array}{c}\text { Raster } \\
\text { Cellular } \\
\text { data }\end{array}$ & $*$ & $*$ & $*$ & Euclidean & Manipulate & $*$ \\
\hline
\end{tabular}

\section{Final comments}

This work shows that each of the four relations is necessary, and defines a set of requirements for supporting simulation of complex spatial systems. We studied the currently available toolkits and verified that they do not support all the requirements

* up to the modeller 
presented in this work. The analysed toolkits use geospatial data for representing space, but only some use it for representing agents. There is no complete support for the four relations, and there is no minimal temporal control. The only way to fulfil these requirements is through a GIS integration; otherwise the toolkit would be limited because there is no other way to be always up-to-dated with the novelties in this area.

Currently, we are developing an ABM extension to TerraME for supporting all the requirements presented in this paper, called TerraME-ABM. TerraME is a toolkit for multiscale modelling of dynamical processes [38]. It is based on Lua [41], one extensible and high-level programming language developed by PUC-Rio, in Brazil. We have as hypothesis that the GPM is a foundation for setting up the relations between the entities of an agent-based model for simulating geospatial phenomena.

To show that each of the four relations is necessary, and that the GPM can model these relations, we have as second objective to develop models using the three relations that involve agents. They are:

Cell $\rightarrow$ agent: We have developed a theoretical model that simulates a competition for space, where agents fight for the cells they belong through a noncooperative game. We propose a definition of mobility, and study how it can affect the results of a given model. This work was submitted to the Journal of Artificial Societies and Social Simulation. The objective here is to build a spatial database with the initial arrangement of the model. With it, the model will only load the arrangement, define the behaviour of the agents, and perform the games.

Agent $\rightarrow$ cell: The centre-north region of the Rondônia state, in Brazil, has its occupation history associated to colonization projects, induced migratory flows, the BR-364 railway construction, and the establishment of development poles [42]. The objective is to study the deforestation trajectory in this region from individual decisions, modelling agents according to their farm size: small and large [43]. There is an early work that uses cellular automata [44]. The objective now is to model agents which decide what to do with their own sets of cells.

Agent $\rightarrow$ agent: The açaí occurs naturally in the Amazonian floodplain forest, providing both fruit and the so-called heart of the palm, or palmito [45, 46]. The objective is to study the açaí economic chain, in Pará, Brazil, exploring questions such as its development and sustainability. This model will be more complex than the other two, once it involves agents in different scales, and it will not be limited to only the agent $\rightarrow$ agent relation.

\section{References}

1. Liu, J., et al., Complexity of coupled human and natural systems. Science, 2007. 317(5844): p. 1513-1516.

2. Cairncross, F., The Death of Distance. 1997, Boston: Harvard Business School Press.

3. Kiesler, S. and J.N. Cummings, What we know about proximity and distance in work groups? A legacy of research, in Distributed Work, P. Hinds and S. Kiesler, Editors. 2002, The MIT Press. p. 57-80. 
4. Robinson, D.T., et al., Comparison of empirical methods for building agent-based models in land use science. Journal of Land Use Science, 2007. 2(1): p. 31-55.

5. Raper, J. and D. Livingstone, Development of a Geomorphological Spatial Model Using Object-Oriented Design. International Journal of Geographical Information Science, 1995. 9(4): p. 359-383.

6. Batty, M., Approaches to Modelling in GIS: Spatial Representation and Temporal Dynamics, in GIS, Spatial Analysis, and Modeling, D. Maguire, M. Batty, and M. Goodchild, Editors. 2005.

7. Leombruni, R. and M. Richiardi, Why are economists sceptical about agent-based simulations? Physica A: Statistical Mechanics and its Applications, 2005. 355(1): p. 103-109.

8. Couclelis, H. Why I No Longer Work with Agents: A Challenge for ABMs of HumanEnvironment Interactions. in Meeting the Challenge of Complexity: Proceedings of the Special Workshop on Agent-Based Models of Land-Use/Land-Cover Change. 2001.

9. Jones, G.T., Agent-based modeling: use with necessary caution. American Journal of Public Health, 2007. 97(5): p. 780-781.

10. Bankes, S.C., Agent-based modeling: A revolution? Proceedings of the National Academy of Sciences, 2002. 99: p. 7199-7200.

11. Bousquet, F. and C. Le Page, Multi-agent simulations and ecosystem management: a review. Ecological Modeling, 2004. 176: p. 313-332.

12. Brian Arthur, W., Out-of-Equilibrium Economics and Agent-Based Modeling, in Handbook of Computational Economics. 2006.

13. Epstein, J.M., Generative social science: studies in agent-based computational modeling. Princeton studies in complexity. 2006, Princeton: Princeton University Press.

14. Tobias, R. and C. Hofmann, Evaluation of free Java-libraries for social-scientific agent based simulation. Journal of Artificial Societies and Social Simulation, 2004. 7(1).

15. Castle, C.J.E. and A.T. Crooks, Principles and Concepts of Agent-Based Modelling for Developing Geospatial Simulations, C.W.P. 110, Editor. 2006, Centre for Advanced Spatial Analysis: London, UK.

16. Railsback, S.F., S.L. Lytinen, and S.K. Jackson, Agent-based Simulation Platforms: Review and Development Recommendations. Simulation, 2006. 82(9): p. 609-623.

17. Brown, D.G., et al., Spatial process and data models: Toward integration of agentbased models and GIS. Journal of Geographical Systems, 2005. 7: p. 25-47.

18. Marietto, M.B., et al., Requirements Analysis of Multi-Agent-Based Simulation Platforms: State of the Art and New Prospects, in Proceedings of Multi-Agent Based Simulation Workshop. 2002: Bologna, Italy.

19. Gasser, L., MAS Infrastructure Definitions, Needs, and Prospects, in Revised Papers from the International Workshop on Infrastructure for Multi-Agent Systems: Infrastructure for Agents, Multi-Agent Systems, and Scalable Multi-Agent Systems. 2000, Springer-Verlag. p. 111 .

20. Gibson, C.C., E. Ostrom, and T.K. Ahn, The concept of scale and the human dimension of global change: a survey. Ecological Economic, 2000. 32: p. 217-239.

21. Tobler, W.R., Cellular Geography, in Philosophy in Geography, S. Gale and G. Ollson, Editors. 1979. p. 379-386.

22. Torrens, P.M., Automata-Based Models of Urban Systems, in Advanced Spatial Analysis: The CASA Book of GIS, P.A. Longley and M. Batty, Editors. 2003, ESRI Press: Redlands, CA. p. 61-81.

23. Torrens, P. and I. Benenson, Geographical automata systems. International Journal of Geographical Information Science, 2005. 19(4): p. 385-412.

24. Benenson, I. and V. Harbash, Object-Based Environment for Urban Simulation: OBEUS User's Guide. 2004, Tel Aviv University.

25. Barber, K.S. and C.E. Martin. Agent autonomy: Specification, measurement, and dynamic adjustment. in Autonomy Control Software Workshop. 1999. Seattle. 
26. Lim, K., et al., Agent-Based Simulation of Household Decision Making and Land Use Change near Altamira, Brazil, in Integrating Geographic Information Systems and AgentBased Modeling Techniques for Simulating Social and Ecological Processes H.R. Gimblett, Editor. 2002, Oxford University Press.

27. Granovetter, M.S., The Strength of Weak Ties. Americal Journal of Sociology, 1973. 78(6): p. 1360-1380.

28. Liben-Nowel, D., et al., Geographic routing in social networks. Proceedings of the National Academy of Sciences, 2005. 102(33): p. 11623-11628.

29. Gastner, M.T. and M.E.J. Newmann, The Spatial Structure of Networks. European Physical Journal B, 2006. 49: p. 247-252.

30. Newmann, M.E.J. and J. Park, Why social networks are different from other types of networks. Physical Review E, 2003. 68(3).

31. Cioffi-Revilla, C., Invariance and universality in social agent-based simulations. Proceedings of the National Academy of Sciences, 2002. 99(3): p. 7314-7316.

32. Minar, N., et al., The Swarm Simulation System: A Toolkit for Building Multi-agent Simulations, in Working Paper. 1997, Santa Fe Institute.

33. Box, P.W., Spatial units as agents: Making the landscape an equal player in agentbased simulations, in Integration of Agent-Based Modelling and Geographic Information Systems, H.R. Gimblett, Editor. 2002, Oxford University Press.

34. North, M., et al. The repast simphony development environment. in Proceedings of the Agent 2005 Conference on Generative Social Processes, Models, and Mechanisms. 2005.

35. Crooks, A.T., The Repast Simulation/Modelling System for Geospatial Simulation. 2007, UCL Centre for Advanced Spatial Analysis.

36. Wilensky, U., NetLogo. 1999, Center for Connected Learning and Computer-Based Modeling, Northwestern University: Evanston, IL.

37. Grigoletti, P.S. and A.C.R. Costa. An Architecture Based on Multi-Agent Systems and Geographic Databases for the Development of Georeferenced Ecological and Social Simulations. in IX Brazilian Symposium on GeoInformatics. 2007. Campos do Jordão, Brazil.

38. Carneiro, T.G.S., Nested-CA: a foundation for multiscale modeling of land use and land change, in Image Processing Division. 2006, INPE: São José dos Campos.

39. Câmara, G., et al. TerraLib: Technology in Support of GIS Innovation. in II Brazilian Symposium on Geoinformatics, GeoInfo2000. 2000. São Paulo.

40. Aguiar, A.P.D., G. Câmara, and R. Cartaxo. Modeling Spatial Relations by Generalized Proximity Matrices. in V Brazilian Symposium in Geoinformatics - GeoInfo 2003. 2003. Campos do Jordão, SP, Brazil.

41. Ierusalimschy, R., Programming in Lua. 2003: Lua.Org.

42. Becker, B.K., Amazônia. 1997, São Paulo, Brazil: Ed. Ática.

43. Escada, M.I.S., et al. Análise de padrões e processos de ocupação para a construção de modelos na Amazônia: Experimentos em Rondônia. in XII Brazilian Symposium on Remote Sensing. 2005.

44. Carneiro, T.G.S., et al., TerraME - A modeling Environment for non-isotropic and non-homogeneous spatial dynamic models development, in LUCC Workshop. 2004: Amsterdam.

45. Brondizio, E.S. and A.D. Siqueira, From Extractivists to Forest Farmers: Changing Concepts of Cabloco Agroforestry in the Amazon Estuary. Research in Economic Anthropology, 1997. 18: p. 233-279.

46. Brondizio, E.S., The Amazonian Caboclo and the Açaí Palm. 2008. 